\title{
ERRATUM
}

\section{Independent and high-level dual-gene expression in adult stem-progenitor cells from a single lentiviral vector}

J Tian and ST Andreadis

Gene Therapy (2012) 19, 957; doi:10.1038/gt.2012.65

Correction to: Gene Therapy (2009) 16, 874-884; doi: 10.1038/ gt.2009.46

Since the publication of this paper, it has been noted that a table was omitted from the Supplementary
Material section. This has now been added to the online Erratum.

The publisher would like to apologize for this error.

Supplementary Information accompanies the paper on Gene Therapy website (http://www.nature.com/gt) 